\title{
Impact of Xpert MTB/RIF assay on multidrug- resistant tuberculosis treatment outcomes in a health district in South Africa
}

\author{
T C Mahwire, ${ }^{1,2} \mathrm{MB} \mathrm{ChB}, \mathrm{MSc}$; M Zunza, ${ }^{3} \mathrm{MSc}, \mathrm{PhD}$; T Marukutira, ${ }^{4,5} \mathrm{MB} \mathrm{ChB}, \mathrm{MPH}$; P Naidoo, ${ }^{6,7} \mathrm{MB} \mathrm{ChB}, \mathrm{MBA}, \mathrm{PhD}$ \\ ${ }^{1}$ HIV and AIDS/STI/TB Department, Port Shepstone Regional Hospital, South Africa \\ ${ }^{2}$ Division of Epidemiology and Biostatistics, Faculty of Medicine and Health Sciences, Stellenbosch University, Cape Town, South Africa \\ ${ }^{3}$ Centre for Evidence-Based Health Care, Faculty of Medicine and Health Sciences, Stellenbosch University, Cape Town, South Africa \\ ${ }^{4}$ Centre for Population Health, Burnet Institute, Melbourne, Australia \\ ${ }^{5}$ School of Public Health and Preventive Medicine, Monash University, Melbourne, Australia \\ ${ }^{6}$ Desmond Tutu TB Centre, Department of Paediatrics and Child Health, Faculty of Medicine and Health Sciences, Stellenbosch University, \\ Cape Town, South Africa \\ Bill and Melinda Gates Foundation, Seattle, Wash., USA
}

Corresponding author: T C Mahwire (tcmahwire@gmail.com)

\begin{abstract}
Background. Xpert MTB/RIF assay rapidly diagnoses rifampicin resistance, enabling early initiation of second-line tuberculosis (TB) treatment. However, the impact of an earlier multidrug-resistant TB (MDR-TB) diagnosis on treatment outcomes is unknown.

Objectives. To compare MDR-TB treatment outcomes in cases diagnosed with smear/culture and Xpert.

Methods. This was a retrospective cohort study with cohorts defined by the diagnostic assay used in presumptive TB cases. Data were extracted from a drug-resistant (DR)-TB register including cases from January 2012 to June 2014. Treatment outcomes were assessed at recorded endpoints or after 2 years for those completing treatment.

Results. A total of 718 cases were enrolled into the study. Cure rates were $43.4 \%(n=158)$ for the smear/culture cohort and $33.5 \%(n=118)$ for the Xpert cohort $(p<0.01)$. Xpert diagnosis (adjusted risk ratio $(\mathrm{aRR}) 0.65 ; p=0.02)$ and male gender $(\mathrm{aRR} 0.66 ; p=0.04)$ were associated with cure outcome. Xpert diagnosis increased time to sputum culture conversion from 4 to 5 months (log-rank test $p=0.01$ ). Time to treatment initiation was not associated with treatment success in logistic regression analysis.

Conclusions. Despite rapid treatment initiation, MDR-TB treatment outcomes were poorer in patients diagnosed with Xpert MTB/RIF assay than in the smear/culture cohort, and they were also poorer in men than in women. Additional studies are required to assess possible factors influencing DR-TB outcomes.
\end{abstract}

S Afr Med J 2019;109(4):259-263. DOI:10.7196/SAMJ. 2019.v109i4.13180

The World Health Organization (WHO) estimates that in 2016, 10.4 million people were infected with tuberculosis (TB) globally, including 1.2 million who were HIV co-infected. ${ }^{[1]}$ Of these, $63 \%$ were notified in TB programmes, reflecting a gap in detection of cases and access to care. ${ }^{[1]}$ In 2014, globally 490000 people had multidrugresistant (MDR)-TB (defined as resistance to both rifampicin and isoniazid), with an additional 100000 rifampicin-monoresistant cases requiring second-line medication. ${ }^{[2]}$ South Africa (SA) faces a huge burden of TB (including MDR-TB) and HIV co-infection. The incidence of TB in 2014 was 834 per 100000 , the TB/HIV co-infection rate was 57\%, and 18734 rifampicin-resistant cases were laboratory confirmed. In total, 11538 patients with rifampicinresistant/MDR-TB were reported to have been started on MDR-TB treatment in SA in the year 2014, reflecting a gap in drug-resistant (DR) TB treatment. ${ }^{[3]}$ Furthermore, many persons with undiagnosed TB were reported to have died from other causes in settings with a high TB burden. ${ }^{[4]}$

Mortality rates of $71 \%$ have been reported in HIV-co-infected MDR-TB patients in KwaZulu-Natal (KZN) Province, SA. ${ }^{[5]}$ Historically, TB has been diagnosed through sputum smear microscopy examination and conventional sputum culture with or without a chest radiograph. Unfortunately, drawbacks of this method include a low sensitivity of smear microscopy in TB/HIV co-infection and a long turnaround time for culture. Long turnaround time leads to inappropriate treatment of MDR-TB patients, with a possible increase of resistance and continued spread of resistant mycobacteria. ${ }^{[6-8]}$ In 2013, the WHO recommended use of Xpert MTB/RIF assay as the initial diagnostic test in presumptive MDR-TB cases and in TB/ HIV co-infection. ${ }^{[7]}$ Xpert MTB/RIF (Xpert) (Cepheid, USA) assay is a fully automated cartridge-based nucleic acid amplification test. A meta-analysis of laboratory and demonstration studies found a pooled sensitivity of $89 \%$ and specificity of $99 \%$, although sensitivity in high HIV prevalence settings is lower, at 79\%. ${ }^{[9]}$ Between 2011 and 2013, the SA National Department of Health rolled out Xpert assays as an initial diagnostic test for all presumptive TB cases to all health districts in the country. ${ }^{[10]}$

Prior to Xpert use, the MDR-TB treatment success rate was $58 \%$ in $\mathrm{KZN},{ }^{[11]}$ which is below the $85 \%$ target set by the WHO's End TB strategy. ${ }^{[12]}$ Studies have reported variable TB treatment outcomes with Xpert. A randomised trial in SA on the effect of replacing microscopy with Xpert found no difference in 6-month mortality in drug-susceptible TB cases. ${ }^{[13]}$

Few studies have addressed the effect of Xpert on MDR-TB patients. One study in SA that compared MDR-TB treatment commencement 
times in line probe assay and Xpert-based algorithms showed a median reduction of 25 days in time to commencement of MDR-TB treatment for patients diagnosed using the Xpert-based algorithm. ${ }^{[2,6]}$ However, the benefit of earlier diagnosis and commencement of appropriate treatment on treatment outcomes is unknown.

\section{Objectives}

The primary objective of the study was to assess whether the use of Xpert as the initial TB diagnostic assay improved MDR-TB treatment outcomes compared with smear/culture/drug-sensitive tests (DSTs) in Ugu Health District, KZN. We hypothesised that the use of Xpert as the primary test in presumptive TB cases would lead to earlier MDR-TB initiation and consequently better clinical outcomes. Our secondary objective was to compare the time to MDR-TB treatment initiation for cases diagnosed by the two methods.

\section{Methods \\ Setting}

The study was undertaken in the Murchison District Hospital DR-TB unit, which serves as the referral centre for all DR-TB patients in Ugu Health District. Ugu Health District had a population of 750000 people and an HIV prevalence rate of $17 \%$ in 2012. ${ }^{[14]}$ All rifampicinresistant and DR-TB patients diagnosed in the district are referred to this unit for DR-TB treatment. The Xpert-based algorithm was phased into the facilities between 2012 and 2013, with variable compliance with the algorithm on the part of clinicians.

\section{Study design}

We retrospectively reviewed medical records of patients with rifampicin-monoresistant and MDR pulmonary TB diagnosed and initiated on MDR-TB treatment between January 2012 and April 2014. Cohorts were defined by the initial diagnostic assay used in testing the presumptive TB case. In the Xpert cohort, the initial diagnostic assay was Xpert. In the smear/culture cohort, the initial diagnostic assay was either smear or culture and DST was via conventional DSTs or the GenoType MTBDRplus (Hain LifeScience $\mathrm{GmbH}$, Germany) line probe assay.

The primary measure of treatment outcome was assessed after a minimum 2-year follow-up period for patients completing 24 months of follow-up or at the clinical endpoint reported for those not completing 24 months of treatment.

\section{Study population}

Inclusion criteria. Patients aged $\geq 15$ years with confirmed pulmonary MDR-TB and rifampicin-monoresistant patients recorded in the DR-TB register at Murchison District Hospital between January 2012 and June 2014 were included in the study.

Exclusion criteria. Patients with monoresistance (other than rifampicin monoresistance), polyresistant TB and extensively drugresistant (XDR)-TB and participants $<15$ years of age (as they are usually not treated at this facility) were excluded from the study.

\section{Treatment outcome definitions}

National Department of Health drug-resistant tuberculosis directorate policy guidelines were used, as the study was a pragmatic examination of the impact of an intervention at a programme level. ${ }^{[10]}$

Cure. A patient with pulmonary $\mathrm{TB}$, with bacteriologically confirmed TB at the start of treatment, who has completed treatment and has converted to smear/culture-negative (with two consecutive negative TB cultures taken 30 days apart). If one positive culture is reported during that time and there is no concomitant clinical evidence of deterioration, a patient may still be considered cured if this positive culture is followed by a minimum of three consecutive negative cultures, taken at least 30 days apart.

Treatment completed. A patient who has completed treatment but does not meet the definition for cure owing to lack of bacteriological results (i.e. fewer than three cultures were negative after TB culture conversion).

Death. A patient who dies from any cause while on DR-TB treatment.

Treatment default. A patient who interrupts DR-TB treatment for 2 or more consecutive months for any reason.

Treatment failure. Treatment is considered to have failed when two or more of the five consecutive cultures taken in the final 12 months are positive, or if any one of the final three cultures is positive. Treatment failure may be observed in patients who do not respond to treatment after 6 - 8 months of effective treatment. Such patients will be put on a different treatment regimen after receiving an outcome of failure and will be allocated to a new treatment cohort.

Transfer out. A patient who has been transferred to a reporting unit in another province and for whom the treatment outcome is unknown.

\section{Ethical considerations}

The Stellenbosch University Human Research Ethics Committee (ref. no. S16/04/079) and the Provincial Health Research and Ethics Committee of KwaZulu-Natal (ref. no. KZ-2016RP32-836) approved the study. De-identified routine medical data were used in the study. Informed consent was waived by the ethics committees because the study used routinely collected historical data and had no direct contact with patients.

\section{Statistical analysis}

We assumed treatment success rates of $50 \%$ in the smear/culture cohort and $60 \%$ in the Xpert cohort, based on previous studies. ${ }^{[15]}$ With a power of $80 \%$ and two-sided alpha of 0.05 , we required 776 participants.

Demographic characteristics and treatment outcomes were compared using the $\chi^{2}$ test or Fisher's exact test. We compared the cumulative probabilities of starting treatment between the Xpert cohort and the smear/culture cohort using Kaplan-Meier estimation methods and the log-rank test. Logistic regression analysis was used to test the influence of covariates on treatment success. Stata 14 (StataCorp, USA) was used for analysis.

\section{Results}

Of a total of 820 MDR-TB cases screened, 718 met the study inclusion criteria (Fig. 1). One hundred and two patients were excluded: 1 was a duplicate entry, 93 had polyresistant TB (not including both isoniazid and rifampicin) and monoresistant $\mathrm{TB}$ (excluding rifampicin monoresistance), and 8 were aged $<15$ years. We enrolled 354 patients into the smear/culture cohort and 364 into the Xpert cohort.

\section{Demographic and clinical characteristics of the study patients}

There were no significant differences between the two groups in terms of gender, age or referral facility (Table 1). The smear/culture cohort had a higher proportion of patients with previously treated $\mathrm{TB}(p<0.01)$. In the smear/culture cohort, 272 of 354 patients $(76.3 \%)$ and in the Xpert cohort 271 of 345 (78.6\%) were HIV-positive. 


\section{MDR-TB treatment outcomes}

In the smear/culture cohort, 158 of 354 patients (43.5\%) were cured compared with 118 of 364 (33.5\%) in the Xpert cohort $(p=0.02)$.

Treatment was completed in 27 of 354 patients $(8.6 \%)$ in the smear/culture cohort compared with 41 of $364(11.7 \%)$ in the Xpert cohort $(p=0.68)$.

In the smear/culture cohort 82 patients $(22.5 \%)$ were lost to follow-up, compared with 100 (28.4\%) in the Xpert cohort $(p<0.01)$ (Table 2).

\section{Predictors of cure outcome}

In multivariable logistic binominal regression analysis, Xpert diagnosis (adjusted risk ratio (aRR) $0.65 ; p=0.02$ ) and male gender (aRR 0.66; $p=0.04$ ) were associated with cure outcome (Table 3 ). Xpert increased time to sputum culture conversion from 4 to 5 months (log-rank test $p=0.01$ ).

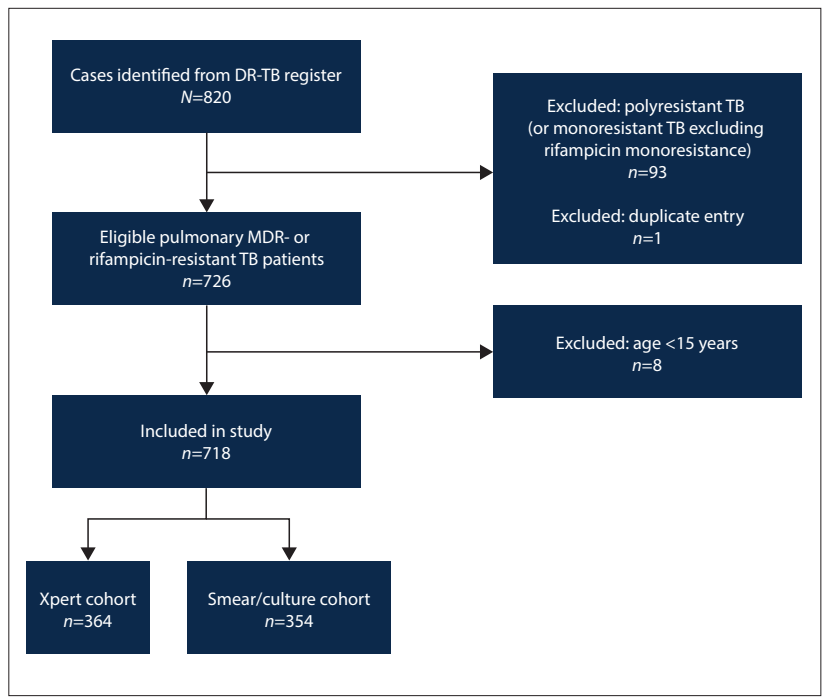

Fig. 1. Cases included in and excluded from the study. (DR = drug-resistant; $T B=$ tuberculosis, $M D R=$ multidrug-resistant. .
Time to treatment initiation was not associated with treatment success in either univariate or multivariate analysis.

\section{Median time to MDR-TB treatment initiation}

Median time to MDR-TB treatment initiation was 62 days (interquartile range (IQR) 56 - 67) in the smear/culture cohort compared with 11 days (IQR 9 - 12) in the Xpert cohort (log-rank test $p<0.01)$ (Fig. 2).

\section{Discussion}

This study compared clinical outcomes between patients diagnosed by smear/culture and those diagnosed by Xpert at the point at which they were presumptive TB cases. The study demonstrated lack of improvement in treatment outcomes in the Xpert cohort, despite a significant reduction in time to treatment initiation in this group. Theoretically, cases diagnosed by Xpert would be diagnosed earlier in their disease progression, and we expected to find improved outcomes. Although treatment delay was not a significant factor for treatment success in the multivariable regression analysis, it should

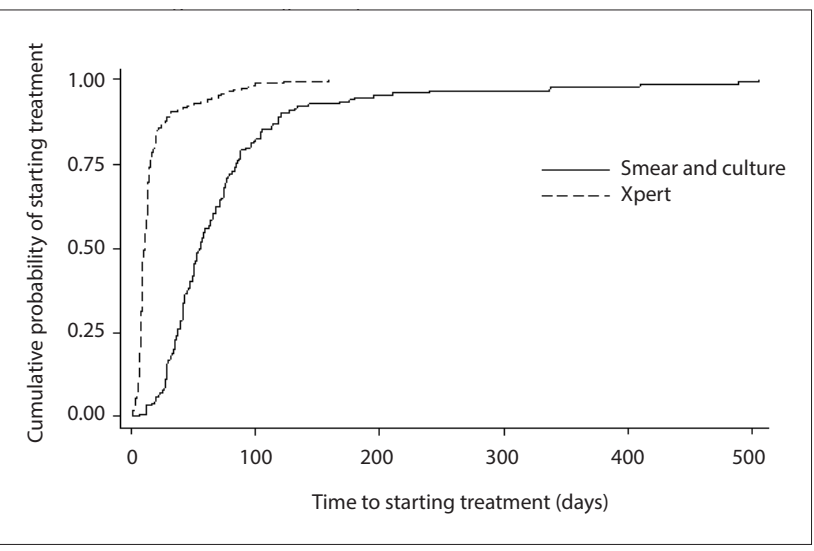

Fig. 2. Comparison of time to MDR-TB treatment initiation in the Xpert and culture cohorts. Log-rank test, $\mathrm{p}<0.01 .(M D R-T B=$ multidrug-resistant tuberculosis.)

Table 1. Demographic and clinical characteristics of study patients

\begin{tabular}{|c|c|c|c|}
\hline Characteristics & Smear/culture cohort $(N=354)$ & Xpert cohort $(N=364)$ & $p$-value \\
\hline Female gender, $n(\%)$ & $185(52.3)$ & $176(48.3)$ & 0.28 \\
\hline Age (years), mean (SD) & $34.8(11.4)$ & $35.0(10.5)$ & 0.83 \\
\hline HIV-positive, ${ }^{\star} n(\%)$ & $271(76.3)$ & $272(78.2)$ & 0.53 \\
\hline TB treatment, $n(\%)$ & & & $<0.01$ \\
\hline New cases & $155(43.8)$ & $238(65.3)$ & \\
\hline Previous first-line TB medication ${ }^{\dagger}$ & $142(40.1)$ & $112(30.8)$ & \\
\hline Previous second-line TB medication & $43(12.2)$ & $13(3.7)$ & \\
\hline ARV history, $n(\%)$ & & & 0.06 \\
\hline On ARVs before treatment started ${ }^{*}$ & $171(63.0)$ & $144(52.8)$ & \\
\hline Started ARVs while on treatment & $84(31.3)$ & $106(39.0$ & \\
\hline Never started ARVs & $15(5.7)$ & $29(8.2)$ & \\
\hline Referral facility, $n(\%)$ & & & 0.20 \\
\hline From primary healthcare $e^{\S}$ & $137(38.8$ & $117(32.3)$ & \\
\hline From district hospital & $33(9.3)$ & $43(11.9)$ & \\
\hline From community health clinic & $170(47.9)$ & $172(47.2)$ & \\
\hline From private institutions & $14(3.9)$ & $6(1.7)$ & \\
\hline
\end{tabular}


Table 2. Comparison of drug-resistant tuberculosis treatment outcomes for the smear/culture and Xpert cohorts

\begin{tabular}{|c|c|c|c|c|c|c|}
\hline Outcome & $\begin{array}{l}\text { Smear/culture cohort } \\
(N=354), n(\%)\end{array}$ & $\begin{array}{l}\text { Xpert cohort }(N=364) \text {, } \\
n(\%)\end{array}$ & $\begin{array}{l}\text { Univariate RR } \\
(95 \% \mathrm{CI})\end{array}$ & $p$-value & $\begin{array}{l}\text { Multivariate aRR } \\
(95 \% \mathrm{CI})\end{array}$ & $p$-value \\
\hline Cured & $158(43.4)$ & $118(33.5)$ & $0.68(0.53-0.87)$ & $<0.01$ & $0.65(0.45-0.92)^{*}$ & 0.02 \\
\hline Treatment completed & $27(8.6)$ & $41(11.7)$ & $1.01(0.60-1.70)$ & 0.98 & $0.28(0.09-0.89)$ & 0.03 \\
\hline Lost to follow-up & $82(22.5)$ & $100(28.4)$ & $1.61(1.14-2.28)$ & $<0.01$ & $\dagger$ & \\
\hline Died & $50(13.7)$ & $61(16.8)$ & $1.08(0.68-1.73)$ & 0.73 & $1.05(0.58-1.9)$ & 0.88 \\
\hline Treatment failure & $35(9.6)$ & $29(8.2)$ & $1.09(0.50-2.38)$ & 0.82 & $0.89(0.34-3.38)$ & 0.87 \\
\hline Transferred out & $2(0.6)$ & $3(0.9)$ & & & & \\
\hline
\end{tabular}

Table 3. Predictors of cure outcome

\begin{tabular}{|c|c|c|c|c|}
\hline Predictor variable & Univariate RR (95\% CI) & $p$-value & Multivariate aRR $(95 \% \mathrm{CI})$ & $p$-value \\
\hline Smear/culture diagnosis & Reference & & & \\
\hline Xpert diagnosis & $0.68(0.53-0.87)$ & $<0.01$ & $0.65(0.45-0.93)$ & 0.02 \\
\hline Age & $1.00(0.99-1.02)$ & 0.44 & $1.01(0.99-1.02)$ & 0.11 \\
\hline Female & Reference & & & \\
\hline Male gender & $0.76(0.59-0.98)$ & 0.04 & $0.66(0.45-0.97)$ & 0.04 \\
\hline Referred from hospital & Reference & & & \\
\hline Referred from community health centre & $0.92(0.58-1.48)$ & 0.76 & $1.14(0.65-2.08)$ & 0.67 \\
\hline Referred from primary healthcare clinic & $1.18(0.89-1.55)$ & 0.25 & $1.19(0.70-1.80)$ & 0.55 \\
\hline Referred from private health institution & $1.07(0.55-2.08)$ & 0.85 & $1.30(0.55-3.04)$ & 0.55 \\
\hline $\mathrm{TB}$ drug treatment naive & Reference & & & \\
\hline Previous first-line TB drug treatment history & $1.04(0.81-1.35)$ & 0.74 & $1.23(0.85-1.77)$ & 0.27 \\
\hline HIV-negative & Reference & & & \\
\hline On ARVs when MDR-TB treatment started & $0.86(0.61-1.14)$ & 0.29 & $1.11(0.78-1.59)$ & 0.55 \\
\hline
\end{tabular}

be noted that we assessed delay only from the time of the first TB test and not from TB symptom onset.

Clinicians chose which assay to use and may have used Xpert on sicker patients, introducing a selection bias that contributed to the lower treatment success rates. It is possible that Xpert assay identified a higher proportion of sicker patients because of its improved sensitivity compared with smear, resulting in the poorer outcomes.

Our study findings were similar to previous studies showing that Xpert assay reduced time to MDR-TB treatment initiation. ${ }^{[16-19]}$ However, early treatment initiation was not associated with treatment success, which may explain the lack of improvement in clinical outcomes. Our study agrees with recent studies demonstrating that use of Xpert has not reduced early mortality in rifampicin-resistant patients. ${ }^{[20]}$

Our study demonstrated poorer outcomes for men than for women, echoing findings from previous studies. ${ }^{[21,22]}$

Previous studies have shown that early initiation of antiretrovirals (ARVs) reduces mortality attributed to TB in HIV co-infected patients. ${ }^{[22,23]}$ Almost half of our patients in the Xpert cohort who were HIV-positive were not on ARV treatment when they were initiated on MDR-TB treatment, compared with $63.0 \%$ in the smear/ culture cohort $(p=0.06)$. However, we were surprised to find that ARV treatment was not significantly associated with treatment success after adjusting for other factors.

\section{Study strengths and limitations}

Strengths of this study were a large sample size and the fact that we included all eligible subjects initiated on MDR-TB treatment in the district during the study period. The study is a pragmatic examination of the impact of an intervention on important clinical endpoints.

Limitations include the retrospective nature of the study using routinely collected data, with some data entries missing. We were unable to control for possible confounders (baseline lung damage, $\mathrm{CD} 4+$ counts, body mass index), as the TB register lacked this clinical information. Our study did not look at initial loss to follow-up in presumptive DR-TB patients, as we only studied those who were diagnosed and entered into care at the DR-TB unit.

For missing outcomes, the primary investigator inferred the outcome from the latest available information in the register using SA national DR-TB guidelines outcome definitions. ${ }^{[10]}$ Patients with missing results were excluded from sub-analysis involving those missing variables, possibly introducing bias.

\section{Implications of the study findings}

Xpert led to early initiation of MDR-TB treatment, which may help reduce further DR-TB transmission. ${ }^{[24]} \mathrm{A}$ recently published study conducted in KZN demonstrated that $69 \%$ of XDR-TB patients had never received MDR-TB treatment, and the XDR-TB was probably due to transmission and not inadequate treatment. The authors concluded that there was a need to interrupt DR-TB transmission. ${ }^{[25]}$

There is a need for interventions targeting men, as they had poorer treatment outcomes than women. However, treatment success rates remain very low in both genders, coupled with high loss to follow-up and mortality. ${ }^{[26]}$ There is a need for further research to determine possible factors associated with treatment success. 


\section{Conclusions}

Despite rapid treatment initiation, MDR-TB treatment outcomes were poorer in patients diagnosed with Xpert MTB/RIF assay than in the smear/culture cohort, and they were also poorer in men than in women. Additional pragmatic studies are required to assess possible factors influencing MDR-TB treatment outcomes.

Declaration. This work was completed as part of TCM's research project towards an MSc (Clinical Epidemiology) degree.

Acknowledgements. The authors thank Stellenbosch University, as this research was conducted as part of the requirements of TCM's MSc course, and the KwaZulu-Natal Department of Health, especially the Murchison District Hospital DR-TB unit for providing access to its DR-TB register. Author contributions. TCM, primary investigator: conception and design of the study, acquisition of data, analysis and interpretation of data, drafting the article, revising it critically for intellectual content, and final approval of the version to be published; PN, supervisor: conception and design, analysis and interpretation of data, drafting the article, revising it critically for intellectual content, and final approval of the version to be published; MZ, biostatistician: analysis and interpretation of data, drafting the article, revising it critically for intellectual content, and final approval of the version to be published; TM, co-author: drafting the article, revising it critically for intellectual content, and final approval of the version to be published.

Funding. This was self-sponsored master's degree research. TCM was assisted with a travel grant from Stellenbosch University to make a short oral presentation at the World Lung Health Conference in Guadalajara, Mexico, in October 2017.

Conflicts of interest. None.

1. World Health Organization. Global Tuberculosis Report 2017. Geneva: WHO, 2017. http://apps.who. int/iris/bitstream/handle/10665/259366/9789241565516-eng.pdf?sequence $=1$ (accessed 9 April 2018). World Health Organization. Multi Drug Resistant Tuberculosis (MDR-TB) 2016 Update. Geneva: WHO, 2016. https://www.who.int/tb/areas-of-work/drug-resistant-tb/en/ (accessed 9 April 2018).

3. National Institute for Communicable Diseases. South African Tuberculosis Drug Resistance Survey . National Institute for Communicable Diseases. South African Tuberculosis Drug Resistance Survey
2012 - 2014. South Africa, 2014. http://www.nicd.ac.za/assets/files/K-12750 NICD National Survey Report_Dev_V11-LR.pdf (accessed 20 December 2017).

Omar T, Variava E, Moroe E, et al. Undiagnosed TB in adults dying at home from natural causes in a high TB burden setting: A post-mortem study. Int J Tuberc Lung Dis 2015;19(11):1320-1325. https:// doi.org/10.5588/ijtld.15.0222

5. Gandhi NR, Shah NS, Andrews JR, et al. HIV coinfection in multidrug- and extensively drug-resistant tuberculosis results in high early mortality. Am J Respir Crit Care Med 2010;181(1):80-96. https://doi. org/10.1164/rccm.200907-0989OC

6. World Health Organization. Guidelines for the Programmatic Management of Multidrug-resistant Tuberculosis. Geneva: WHO, 2011. https://www.who.int/tb/challenges/mdr/programmatic guidelines_for_mdrtb/en/ (accessed 9 April 2019).
7. World Health Organization. Xpert MTB/RIF Implementation Manual: Technical and Operational 'How-to': Practical Considerations. Geneva: WHO, 2014. https://apps.who.int/iris/bitstream/ How-to: Practical Considerations. Geneva: WHO, 2014. https://apps.who.int/iris/bitstream/
handle/10665/112469/9789241506700_eng.pdf;jsessionid=749CC4FC08848FBB9534537521F02415?

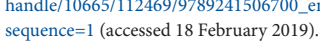

8. Balcha TT, Sturegård $\mathrm{E}$, Winqvist N, et al. Intensified tuberculosis case-finding in HIV-positive adults
since managed at Ethiopian health centers: Diagnostic yield of Xpert MTB/RIF compared with smear microscopy and liquid culture. PLoS One 2014;9(1). https://doi.org/10.1371/journal.pone.0085478

9. Steingart KR, Schiller I, Horne DJ, Pai M, Boehme CC, Dendukuri N. Xpert ${ }^{*}$ MTB/RIF assay for pulmonary tuberculosis and rifampicin resistance in adults. Cochrane Database Syst Rev 2014, Issue 1. Art. No. CD009593. https://doi.org/10.1002/14651858.CD009593.pub3

10. National Department of Health, South Africa. Management of drug-resistant tuberculosis: Policy guidelines (updated - January 2013). South Africa, 2013. http://www.health-e.org.za/wp-content/ guidelines (updated - January 2013). South Africa, 2013. http://www.health-e.org.za/wp-content/

uploads/2014/06/MDR-TB-Clinical-Guidelines-Updated-Jan-2013.pdf (accessed 18 February 2019).
11. Loveday M, Wallengren K, Brust J, et al. Community-based care vs. centralised hospitalisation for MDR-TB patients, KwaZulu-Natal, South Africa. Int J Tuberc Lung Dis 2015;19(2):163-171. https:// MDR-TB patients, KwaZulu-
doi.org/10.5588/ijtld.14.0369

12. Uplekar M, Stop TB Partnership and World Health Organization. The Stop TB strategy: Building On and Enhancing DOTS to Meet the TB-related Millennium Development Goals. Geneva: World Healt Organization, 2006. http://www.who.int/iris/handle/10665/69241 (accessed 18 February 2019).

13. Churchyard GJ, Stevens WS, Mametja LD, et al. Xpert MTB/RIF versus sputum microscopy as the initial diagnostic test for tuberculosis: A cluster-randomised trial embedded in South African rollout of Xpert MTB/RIF. Lancet Glob Health 2015;3(8):e450-e457. https://doi.org/10.1016/S2214109X(15)00100-X

14. South African National AIDS Council. National Strategic Plan on HIV, STIs and TB 2012 - 2016. http://www.sanac.org.za/nsp/the-national-strategic-plan (accessed 27 September 2014).

15. Loveday M, Padayatchi N, Wallengren K, et al. Association between health systems performance and treatment outcomes in patients co-infected with MDR-TB and HIV in KwaZulu-Natal, South Africa: Implications for TB programmes. PLoS One 2014;9(4):e94016. https://doi.org/10.1371/journal. pone. 0094016

16. Theron G, Zijenah L, Chanda D, et al. Feasibility, accuracy, and clinical effect of point-of-care Xpert MTB/RIF testing for tuberculosis in primary-care settings in Africa: A multicentre, randomised, controlled trial. Lancet 2014;383(9915):424-435. https://doi.org/10.1016/S0140-6736(13)62073-5

17. Mupfumi L, Makamure B, Chirehwa M, et al. Impact of Xpert MTB/RIF on antiretroviral therapyassociated tuberculosis and mortality: A pragmatic randomized controlled trial. Open Forum Infect Dis 2014;1(1):ofu038. https://doi.org/10.1093/ofid/ofu038

18. Iruedo J, O'Mahony D, Mabunda S, Wright G, Cawe B. The effect of the Xpert MTB/RIF test on the time to MDR-TB treatment initiation in a rural setting: A cohort study in South Africa’s Eastern Cape Province. BMC Infect Dis 2017;17(1):91. https://doi.org/10.1186/s12879-017-2200-8

19. Umanah T, Ncayiyana J, Padanilam X, et al. Treatment outcomes in multidrug resistant tuberculosishuman immunodeficiency virus co-infected patients on anti-retroviral therapy at Sizwe Tropical Disease Hospital Johannesburg, South Africa. BMC Infect Dis 2015;15(1):478. https://doi.org/10.1186/ s12879-015-1214-3

20. Schnippel K, Firnhaber C, Ndjeka N, et al. Persistently high early mortality despite rapid diagnostics for drug-resistant tuberculosis cases in South Africa. Int J Tuberc Lung Dis 2017;21(10):1106-1111. https://doi.org/10.5588/ijtld.17.0202

21. Maskew M, Brennan AT, Westreich D, McNamara L, MacPhail AP, Fox MP. Gender differences in mortality and CD4 count response among virally suppressed HIV-positive patients. J Womens Health (Larchmt) 2013;22(2):113-120. https://doi.org/10.1089/jwh.2012.3585

22. Feng J-Y, Huang S-F, Ting W-Y, et al. Gender differences in treatment outcomes of tuberculosi patients in Taiwan: A prospective observational study. Clin Microbiol Infect 2012;18(9):E331-E337 https://doi.org/10.1111/j.1469-0691.2012.03931.x

23. INSIGHT START Study Group. Initiation of antiretroviral therapy in early asymptomatic HIV infection. N Engl Med J 2015;373(9):795-807. https://doi.org/10.1056/NEJMoa1506816

24. Dharmadhikari AS, Mphahlele M, Venter K, et al. Rapid impact of effective treatment on transmission of multidrug-resistant tuberculosis. Int J Tuberc Lung Dis 2014;18(9):1019-1025. https;//doi. org $/ 10.5588$ /ijtld. 13.0834

25. Shah NS, Auld SC, Brust JCM, et al. Transmission of extensively drug-resistant tuberculosis in South Africa. N Engl J Med 2017;376(3):243-253. https://doi.org/10.1056/NEJMoa1604544

26. Auld AF, Fielding KL, Gupta-Wright A, Lawn SD. Xpert MTB/RIF - why the lack of morbidity and mortality impact in intervention trials? Trans R Soc Trop Med Hyg 2016;110(8):432-444. https://do. org/10.1093/trstmh/trw056

Accepted 7 September 2018 